\title{
Regulated Pumilio-2 binding controls RINGO/Spy mRNA translation and CPEB activation
}

\author{
Kiran Padmanabhan and Joel D. Richter ${ }^{1}$ \\ Program in Molecular Medicine, University of Massachusetts Medical School, Worcester, Massachusetts 01605, USA
}

CPEB is a sequence-specific RNA-binding protein that controls the polyadenylation-induced translation of mos and cyclin B1 mRNAs in maturing Xenopus oocytes. CPEB activity requires not only the phosphorylation of S174, but also the synthesis of a heretofore-unknown upstream effector molecule. We show that the synthesis of RINGO/Spy, an atypical activator of cyclin-dependent kinases (cdks), is necessary for CPEB-directed polyadenylation. Deletion analysis and mRNA reporter assays show that a cis element in the RINGO/Spy $3^{\prime}$ UTR is necessary for translational repression in immature (G2-arrested) oocytes. The repression is mediated by 3'UTR Pumilio-Binding Elements (PBEs), and by its binding protein Pumilio 2 (Pum2). Pum2 also interacts with the Xenopus homolog of human Deleted for Azoospermia-like (DAZL) and the embryonic poly(A)-binding protein (ePAB). Following the induction of maturation, Pum2 dissociates not only from RINGO/Spy mRNA, but from XDAZL and ePAB as well; as a consequence, RINGO/Spy mRNA is translated. These results demonstrate that a reversible Pum2 interaction controls RINGO/Spy mRNA translation and, as a result, CPEB-mediated cytoplasmic polyadenylation.

[Keywords: Pumilio-2; translation; Xenopus oocytes]

Received October 11, 2005; revised version accepted November 17, 2005.

In vertebrate oocytes, meiotic maturation is a hormonetriggered reduction division that is necessary to produce haploid eggs in preparation for fertilization (Ferrell 1999). The preceding period of oocyte growth is characterized by the synthesis and storage of many macromolecules that are destined for use not only during maturation, but also during the early embryonic cleavage divisions that occur in the absence of transcription. In Xenopus, fully grown oocytes are arrested in prophase of meiosis I (MI), a state that resembles G2 of the somatic cell cycle; in response to progesterone acting at or near the cell surface, the oocytes enter the meiotic division phase. This process requires the activation of several protein kinases including the mitogen-activated protein kinase (MAPK) and cyclin-dependent kinase 1 (cdk1), which when associated with the cofactor cyclin B1 is referred to as maturation-promoting factor (MPF). Several preceding and concomitant events are also necessary for maturation. One of these is the translation of dormant mos mRNA (Sheets et al. 1994); Mos activates a MAPK cascade and indirectly induces the translation of cyclin B1 mRNA (Ballantyne et al. 1997; de Moor and Richter 1997). Mos (and cyclin) mRNA translation is regulated by cytoplas-

${ }^{1}$ Corresponding author.

E-MAIL Joel.richter@umassmed.edu; FAX (508) 856-4289.

Article and publication are at http://www.genesdev.org/cgi/doi/10.1101/ gad.1383106. mic polyadenylation, which in turn requires the 3'UTRresiding cytoplasmic polyadenylation element (CPE) (Paris et al. 1991; Sheets et al. 1994). The CPE is bound by CPEB (Hake and Richter 1994), a protein whose polyadenylation-stimulatory activity is controlled by Aurora A-catalyzed phosphorylation (Mendez et al. 2000). CPEB must interact with several factors to stimulate polyadenylation; these include (1) symplekin, a protein that seems to act as a scaffold upon which other proteins are assembled; (2) CPSF (cleavage and polyadenylation specificity factor), a group of four proteins that also bind another essential cis element, the hexanucleotide AAUAAA; and (3) Gld2, a cytoplasmic poly(A) polymerase (Mendez et al. 2000; Barnard et al. 2004). Yet another factor bound to CPEB, Maskin is probably the most proximal one that regulates translation. Maskin, while anchored to specific RNAs through CPEB, also binds the cap-binding factor eIF4E; the latter association prevents the formation of the eIF4F (eIF4A, eIF4E, eIF4G) initiation complex. Translational activation is triggered by CPEB-stimulated poly(A) tail growth and the recruitment of the poly(A)-binding protein (PABP)-eIF4G complex, which displaces Maskin from eIF4E and positions the 40s ribosomal subunit on the 5 '-end of the mRNA (Stebbins-Boaz et al. 1999; Cao and Richter 2002; Richter and Sonenberg 2005). Maskin also undergoes several phosphorylation events during this time that also help it dissociate from eIF4E (Barnard et al. 2005). 
There are several other processes that precede, and in some cases, are necessary for polyadenylation, one of which is a signaling cascade leading to the abrogation of GSK3 $\beta$-mediated inhibition of Aurora A (Sarkissian et al. 2004). Another is a protein synthesis requirement for CPEB-dependent polyadenylation. That is, culturing oocytes in cycloheximide (CHX) completely abrogates CPE-mediated polyadenylation. However, CHX treatment does not have a similar effect on oocytes that have already reinitiated meiosis for $\sim \mathrm{h}$ (McGrew and Richter 1990). Moreover, the block to polyadenylation can be reversed by the microinjection of recombinant cyclin B1, which presumably activates free cdks (Paris et al. 1991). In addition to polyadenylation, cdk activity is also necessary for the activation of the Mos-MAPK and MPF pathways (Nebreda et al. 1995). Thus, it seemed possible that a small amount of cyclin B1 mRNA was translated very soon after progesterone administration, and that the encoded protein induced some cdk activity that, in turn, stimulated CPEB-mediated polyadenylation.

Ferby et al. (1999) devised a screen to identify factors that would quickly stimulate maturation after injection into oocytes; their regimen led to the isolation of RINGO (Rapid Inducer of G2-M in Oocytes). In addition, a genetic screen in fission yeast to identify cell cycle regulators of the G2-M transition resulted in the discovery of a RINGO homolog called Speedy (Spy) (Lenormand et al. 1999/. Overexpression of RINGO/Spy in oocytes rapidly induced Mos synthesis, MAPK and MPF activation, and maturation. Conversely, these events were blocked or delayed if progesterone-treated oocytes were injected with antisense oligodeoxynucleotides (AS ODN) against RINGO/Spy mRNA (Ferby et al. 1999; Lenormand et al. 1999). RINGO/Spy binds to and activates cdk1 (and cdk2) as well as associates with p27, the cdk inhibitor, indicating its molecular function in cell cycle regulation (Karaiskou et al. 2001; Porter et al. 2003; Dinarina et al. 2005). RINGO/Spy may associate with free cdk1 in oocytes and eventually trigger MPF activation and maturation. Moreover, such results suggest that the translation of RINGO/Spy mRNA might be an early and critical event for the activation of CPEB.

We have investigated not only the importance of RINGO/Spy protein for CPEB-mediated polyadenylation, but also the mechanism by which RINGO/Spy mRNA translation is controlled. We find that RINGO/ Spy synthesis precedes and is essential for CPEB phosphorylation and all resulting downstream effects including mos mRNA polyadenylation and translation. In immature oocytes, a region of the 3'UTR that includes Pumilio 2-Binding Elements (PBEs) represses RINGO/ Spy mRNA translation. The PBE is bound by Pum2, which also associates with Deleted for Azoospermia-like (DAZL), an RNA-binding protein (Cooke and Elliott 1997), and embryonic PABP (ePAB), an embryonic form of the poly(A)-binding protein (Voeltz et al. 2001). The injection of oocytes with either Pum 2 antibody or a portion of Pum2 that acts as a dominant-negative form of the protein stimulates RINGO/Spy synthesis and oocyte maturation, thus demonstrating the importance of Pum2 for translational inhibition. Upon progesterone treatment of oocytes, Pum2 dissociates not only from RINGO/Spy RNA, but from DAZL and ePAB as well; the consequence of this dissociation is RINGO/Spy mRNA translation. These results show that Pum2-mediated translation of RINGO/Spy mRNA is required for CPEB activation.

\section{Results}

RINGO/Spy is necessary for CPEB-mediated polyadenylation in oocytes

While Xenopus oocytes have no detectable RINGO/SPY protein, they do contain RINGO/Spy mRNA whose translation is essential for the completion of maturation (Ferby et al. 1999). Because CPEB-mediated polyadenylation and translation of mos mRNA is an early and necessary event for maturation, we sought to determine whether RINGO/Spy mRNA translation precedes or is subsequent to this process. Oocytes were injected with an antisense oligodeoxynucleotide against RINGO/Spy mRNA (RINGO AS ODN), which Ferby et al. (1999) showed specifically induced the destruction of this mRNA and inhibited maturation, followed by a radiolabeled CPE-containing mos RNA. The oocytes were stimulated with progesterone, and the RNA was subsequently extracted and analyzed for polyadenylation by urea-PAGE. As expected, progesterone induced polyadenylation, detected as reduced mobility of the RNA probe, in both mock-injected oocytes and in oocytes injected with a control AS ODN (Fig. 1A, lanes 3,7). However, injection of the RINGO/Spy AS ODN resulted in a near complete prevention of this process (Fig. 1A, lane 5). RTPCR analysis showed that injection of the RINGO/Spy AS ODN, but not the control ODN, resulted in RINGO/ Spy RNA destruction (Fig. 1A, bottom). Furthermore, the RINGO/Spy AS ODN prevented Mos synthesis when assessed either by Western blotting for this protein (Fig. 1B, lanes $4,6,8)$, or by ${ }^{35}$ S-methionine metabolic labeling and immunoprecipitation (IP) (Fig. 1C, lanes 4-7). Thus, RINGO/Spy is required for polyadenylation and translation of mos mRNA.

Aurora A-catalyzed CPEB S174 phosphorylation is necessary to activate the polyadenylation machinery (Mendez et al. 2000). To determine whether RINGO/Spy is necessary for this event, oocytes were injected with RINGO/Spy AS ODN as before, followed by ${ }^{32} \mathrm{P}$ labeling and CPEB IP. While CPEB was ${ }^{32}$ P-phosphorylated soon after progesterone treatment, as expected, the labeling was almost completely prevented by the RINGO/Spy AS ODN (Fig. 1D, lanes 5-8). At $6 \mathrm{~h},{ }^{32}$ P-labeling of CPEB correlated with Mos synthesis (Fig. 1D, lane 9), which specifically requires S174 phosphorylation (Mendez et al. 2000). These data indicate that RINGO/Spy mRNA translation precedes and is necessary for CPEB activation. We have also confirmed these results by first injecting mos AS ODN into oocytes prior to progesterone treatment; while not able to complete maturation (Sagata et al. 1988), the hormone still activated CPEB and 

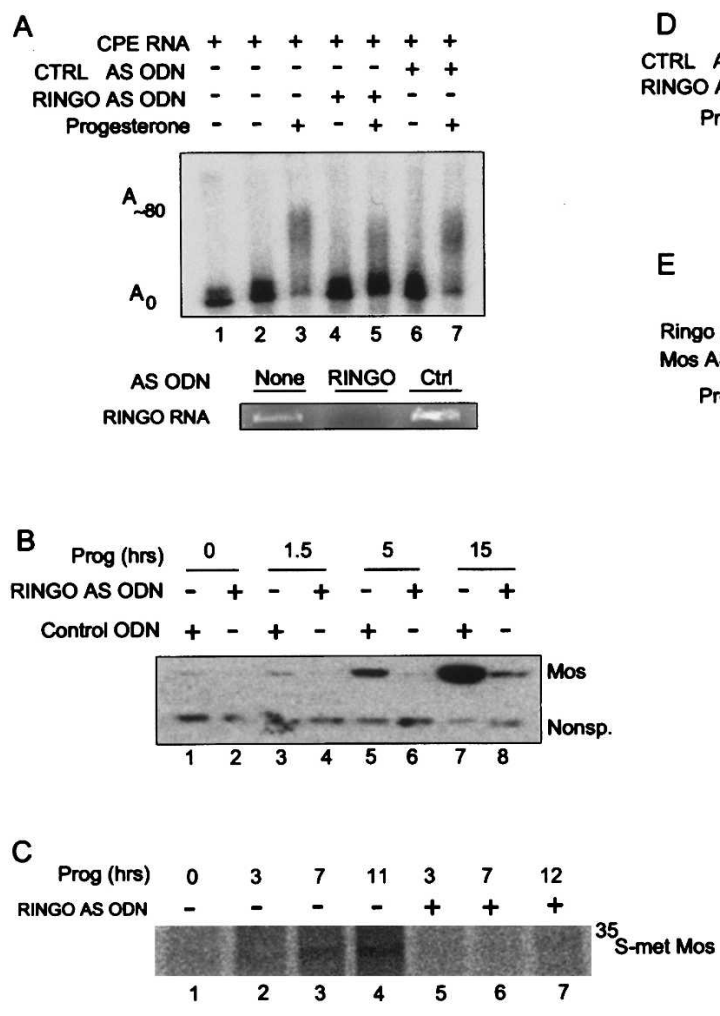

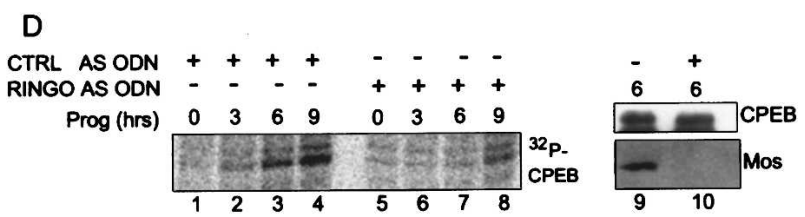

E

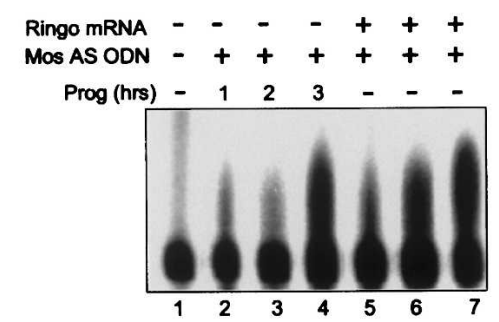

F

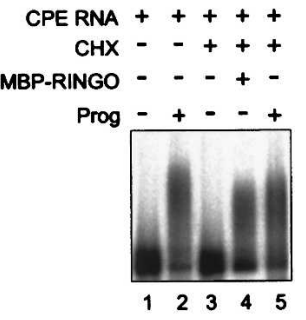

G

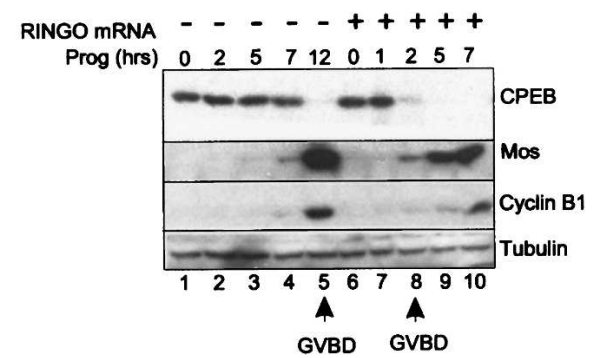

Figure 1. RINGO/Spy is necessary for CPEB-mediated polyadenylation. (A) Oocytes were injected with buffer only, AS ODN against RINGO/Spy RNA, or a control nonspecific AS ODN and incubated for $5 \mathrm{~h}$ followed by the injection of a radiolabeled CPE-containing mos RNA. Following a further incubation for $6 \mathrm{~h}$ in the absence or presence of progesterone, total RNA was extracted and analyzed for polyadenylation by denaturing polyacrylamide gel electrophoresis. (Lower panel) The efficiency of the destruction of RINGO/Spy mRNA by RINGO/Spy and control AS ODNs was assessed by RT-PCR. (B) The RINGO/Spy and control AS ODNs were injected into oocytes followed by a time course of progesterone treatment; Mos was then detected by Western blotting. By $10 \mathrm{~h}, 50 \%$ of the oocytes injected with the control AS ODN had matured. $(C)$ Oocytes were either mock-injected or injected with RINGO/Spy AS ODN and incubated in medium containing ${ }^{35} \mathrm{~S}$-methionine $/{ }^{35} \mathrm{~S}$-cysteine. Following progesterone stimulation, groups of 100 oocytes were collected at various time points, lysed, and Mos was immunoprecipitated and analyzed by SDS-PAGE. $(D)$ Oocytes were injected with control or RINGO/Spy AS ODNs and $\left[\gamma^{3}{ }^{32} \mathrm{P}\right]$ ATP. Following incubation in progesterone, groups of 50 oocytes were lysed at several time points, and CPEB was immunoprecipitated and analyzed by SDS-PAGE. (Right) The lysates were also probed on Western blots for Mos and CPEB. Fifty percent of the oocytes matured by $11 \mathrm{~h} .(E)$ Oocytes were injected with a mos AS ODN and radiolabeled CPE-containing mos 3'UTR. Following a 5-h incubation, the oocytes were mock-injected or injected with an mRNA encoding MBP-tagged RINGO/Spy. After a further 3-h incubation, the mock-injected oocytes were stimulated with progesterone and collected every hour for $3 \mathrm{~h}$. Oocytes injected with MBP-RINGO mRNA, which was not treated with progesterone, were harvested in parallel and processed as in $A$. (F) Oocytes injected with CPE-containing RNA were treated with progesterone (lane 2); in some cases, they were transferred to Barth's medium containing $10 \mu \mathrm{g} / \mathrm{mL}$ CHX $1 \mathrm{~h}$ after hormone treatment (lane 5). Other oocytes cultured in the presence of CHX were injected with CPE-containing RNA; some were also injected with MBP-RINGO/Spy protein and cultured for $6 \mathrm{~h}$ (lane 4) before the RNA was analyzed as in $A$. (G) Immunoblot analysis for CPEB, cyclin B1, Mos, and tubulin from oocytes treated with progesterone only or injected with mRNA (15 $\mathrm{nL}$ of $0.5 \mu \mathrm{g} / \mu \mathrm{L}$ mRNA) encoding MBP-RINGO/Spy prior to progesterone treatment.

stimulated the polyadenylation of injected mos RNA (Fig. 1E, lanes 2-4; see also de Moor and Richter 1997). Strikingly, oocytes that were injected with RINGO/Spy mRNA stimulated polyadenylation of mos RNA even in the absence of progesterone (Fig. 1E, lanes 5-7). To determine whether RINGO/Spy was sufficient for inducing polyadenylation when protein synthesis was inhibited, oocytes cultured in medium containing $10 \mu \mathrm{g} / \mathrm{mL}$ CHX were injected with CPE-containing RNA and MBPRINGO/Spy protein. MBP-RINGO/Spy stimulated polyadenylation in these oocytes to the same extent as mock- injected non-CHX-treated oocytes or oocytes cultured in CHX following a 1-h treatment of progesterone (Fig. 1F, lanes 2,4,5). Finally, oocytes were also injected with RINGO/Spy mRNA followed by progesterone treatment; not only did RINGO/Spy accelerate maturation (germinal vesicle breakdown, or GVBD; 2 vs. $12 \mathrm{~h}$ for those oocytes not injected with RINGO/Spy mRNA), but it also accelerated CPEB destruction (Mendez et al. 2002), and Mos and cyclin B1 synthesis (Fig. 1G). Thus, by these criteria, RINGO/Spy mRNA translation precedes and is necessary for probably all CPEB-mediated processes. 


\section{A 3'UTR cis element regulates translation of RINGO/Spy mRNA}

To directly investigate the translation of RINGO/Spy mRNA during oocyte maturation, antibody against fulllength recombinant protein was generated and used for IP experiments from immature and mature oocytes that were metabolically labeled with ${ }^{35} \mathrm{~S}$-methionine $/{ }^{35} \mathrm{~S}$-cysteine. While a prominent band corresponding in size 140 $\mathrm{kDa}$ ) to RINGO/Spy was immunoprecipitated from oocytes treated with progesterone, several other bands were also evident, including one that might be a product of RINGO/Spy degradation (Fig. 2A, asterisk; Ferby et al. 1999). To confirm that the indicated band was indeed RINGO/Spy, the experiment was repeated with oocytes injected with RINGO/Spy AS ODN; in this case the 40$\mathrm{kDa}$ band was not detected (Fig. 2A, lanes 4-6). Thus,
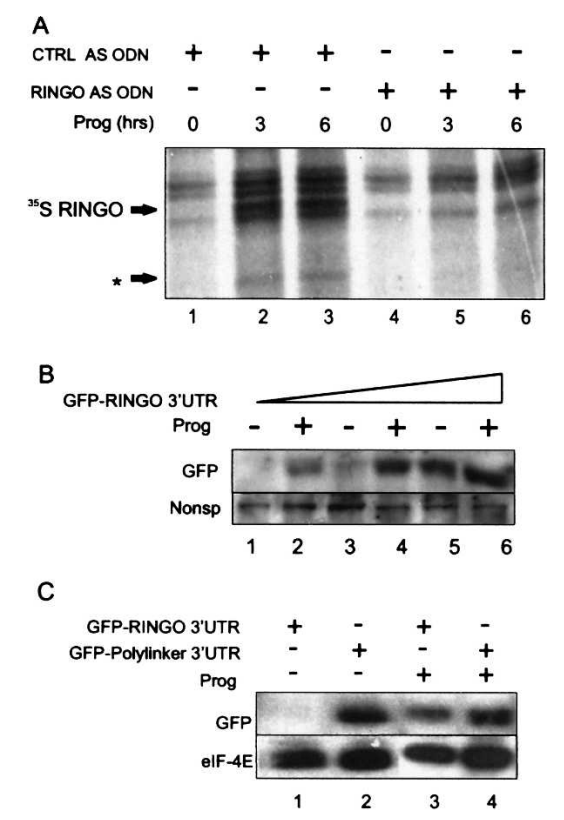

Figure 2. RINGO/Spy mRNA is under translational control. (A) Oocytes injected with control or RINGO/Spy AS ODNs were incubated overnight in medium containing ${ }^{35} \mathrm{~S}$-methionine $/{ }^{35}$ S-cysteine and then treated with progesterone. Several times thereafter, the oocytes were lysed and used for RINGO/ Spy IP. The arrows indicate two proteins that are detected in control AS ODN-injected oocytes during maturation that are absent in the RINGO AS ODN-injected oocytes. The top band corresponds in size to full-length RINGO/Spy, while the one denoted by an asterisk corresponds in size to a putative RINGO/ Spy breakdown product (Ferby et al. 1999). (B) Oocytes were injected with increasing concentrations of mRNA encoding GFP fused to the RINGO/Spy 3'UTR $(9,18$, and $32 \mathrm{~nL}$ of a 10 $\mathrm{pg} / \mathrm{nL}$ RNA solution) and in some cases, were treated with progesterone. Lysates from the oocytes were analyzed for GFP by Western blotting. A nonspecific protein that reacted with the GFP antibody served as a loading control. (C) Oocytes were injected with mRNA encoding GFP fused to the RINGO/Spy 3'UTR or a polylinker sequence. After overnight incubation, the oocytes were stimulated with progesterone, lysed, and immunoblotted for GFP and eIF4E, which served as a control. dormant RINGO/Spy mRNA is translated soon after progesterone treatment.

To investigate RINGO/Spy mRNA translational control, we focused on the 3'UTR, which often contains regulatory signals (Wickens et al. 2002), and fused it to GFP. Three concentrations of the RNA were injected into oocytes, some of which were treated with progesterone. At the lowest concentration (0.08 ng/oocyte), GFP-RINGO/Spy-3'UTR RNA was repressed in oocytes but translated after maturation (Fig. 2B, lanes 1,2). In contrast, GFP-RINGO/Spy-3'UTR RNA was translated in oocytes when injected at the highest concentration $(0.35 \mathrm{ng} /$ oocyte) even in the absence of progesterone (Fig. $2 \mathrm{~B}$, lanes 5,6$)$; the middle concentration $(0.15 \mathrm{ng} /$ oocyte) gave an intermediate response (Fig. 2B, lanes 3,4). Thus, the lowest concentration most accurately reflects the endogenous RINGO/Spy RNA translation pattern and was used for all subsequent experiments.

In addition to the RINGO/Spy 3'UTR, GFP was appended with a polylinker sequence, which presumably is devoid of all regulatory information. This $3^{\prime} \mathrm{UTR}$ had little effect on robust GFP RNA translation in either control or mature oocytes (Fig. 2C, cf. lanes 1,2 and 3,4). Therefore, the RINGO/Spy 3'UTR confers translational silencing in oocytes.

To define a minimal sequence in the RINGO/Spy 3'UTR that was responsible for translational silencing, a series of deletion mutants was constructed within this region that were then appended to GFP RNA; following oocyte injection, GFP synthesis was examined by Western blotting (Fig. 3A). While deletion of the $5^{\prime}$-most 178 nucleotides (nt) of the 3'UTR had little effect on GFP synthesis, removal of 44 nt near the $3^{\prime}$ terminus [less the poly(A) tail] had a dramatic effect; this mutant, $\Delta 6$, was translated nearly as well in immature oocytes as a GFP RNA containing the full RINGO/Spy 3'UTR was translated after progesterone treatment (Fig. 3B).

Visual inspection of the $\Delta 6$ repressor element revealed the presence of two putative binding sites for Pumilio 2 (Pum2) (Fig. 3A, boxed sequences). These sites, henceforth referred to as the PBEs were identified initially by in vitro SELEX (systematic evolution of ligands by exponential enrichment) experiments to distinguish them from the Nanos response element (NRE), which is bound by Pumilio 1 (Pum1). While both the PBE and the NRE contain essential UGUA motifs, the NRE is a bipartite sequence consisting of a 5' Box A (GUUGU) and a 3' Box B (UGUA) (Zamore et al. 1999). The PBE dispenses with Box A and instead consists of a UGUANAUA sequence (White et al. 2001). The RINGO/Spy 3'UTR $\Delta 6$ region contains two putative PBEs, UGUAUAAA and UGUAAAUA, $55 \mathrm{nt}$ and $65 \mathrm{nt}$ from the $3^{\prime}$ end of the mRNA.

\section{Pum2 interacts with the RINGO/Spy 3'UTR and represses translation}

To investigate whether Pum2 is capable of binding the putative PBEs in the RINGO/Spy 3'UTR, the RNA-bind- 


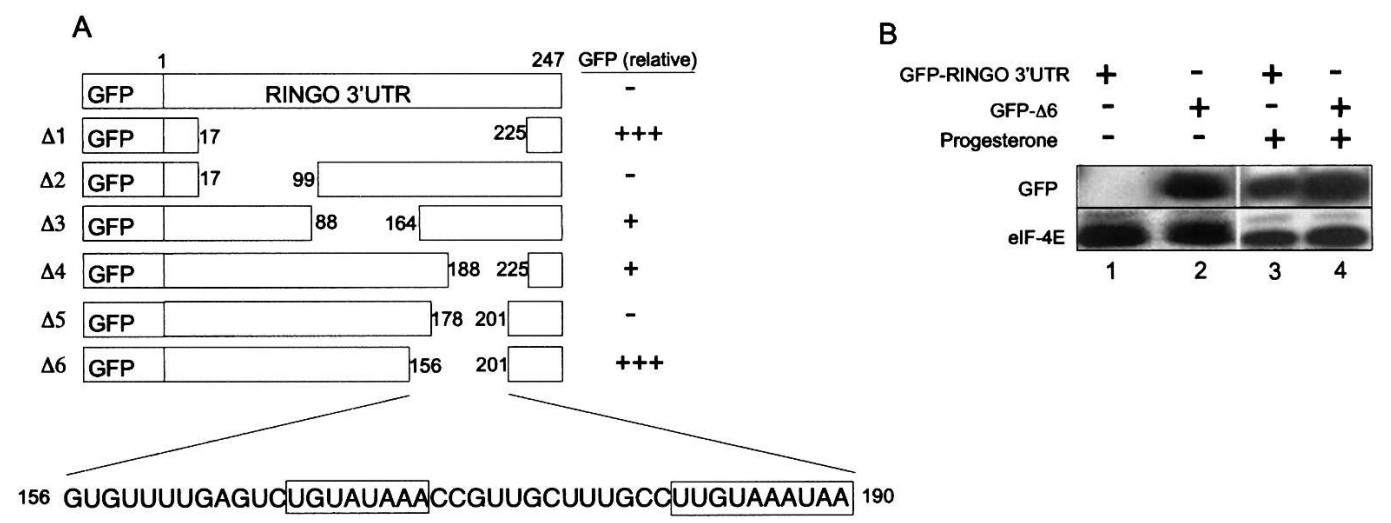

Figure 3. PBEs regulate RINGO mRNA translation. (A) Several deletions of the RINGO/Spy 3'UTR were constructed and appended to the GFP-coding region. The corresponding RNAs were injected into oocytes that were then immunoblotted for GFP. The relative amount of GFP produced from each construct is indicated. The $\Delta 6$ construct lacked $44 \mathrm{nt}$, which are indicated at the bottom. The boxed regions refer to putative PBEs. (B) The level of GFP synthesis when the RNA was appended with either the full-length RINGO/Spy 3'UTR or the $\Delta 6$ 3'UTR.

ing portion of Pum2, the PUF region (Pumilio-FBF; FBF is an RNA-binding protein originally identified in Caenorhabditis elegans [Wickens et al. 2002]; hence the "PUF region" refers to the RNA-binding portion of the protein) was expressed in Escherichia coli, purified, and used in a gel mobility-shift assay. This protein slowed the electrophoretic mobility of the RINGO/Spy 3'UTR; the mobility shift was blocked by the addition of excess unlabeled 3'UTR but not by the addition of unlabeled excess 3 'UTR $\Delta 6$ (Fig. 4A). Thus, Pum2 most probably binds the PBEs within the $46 \mathrm{nt}$ of the RINGO/Spy 3'UTR that are deleted from the $\Delta 6$ region.

Pum2 is present in oocytes both before and after maturation (Fig. 4B). To determine whether oocyte Pum 2 can bind the RINGO/Spy 3'UTR, a ${ }^{32}$ P-labeled probe encompassing this region, as well as one corresponding to $3^{\prime} \mathrm{UTR} \Delta 6$, was added to an oocyte extract that was irradiated with UV light. The extract was then digested with RNase $\mathrm{A}$ and $\mathrm{T} 1$, and the proteins made radioactive by label transfer were displayed on an SDS gel. One band, corresponding to the precise size of Pum2, as assessed by Western blotting, crosslinked to the full-length RINGO/Spy 3'UTR, but not the $\Delta 6$ 3'UTR (Fig. 4B). Therefore, endogenous Pum2 can bind a region of the RINGO/Spy 3'UTR that contains PBEs.

To determine whether Pum2 represses RINGO/Spy mRNA translation, an antibody generated against the $\mathrm{N}$ terminus of Pum2 was injected into oocytes that had previously been injected with mRNAs encoding GFP fused to the full-length RINGO/Spy 3'UTR. This antibody, but not a nonspecific IgG, induced the translation of GFP RNA (Fig. 4C). We next tested whether the antibody could induce the synthesis of endogenous RINGO/ Spy protein. Following Pum 2 antibody or nonspecific IgG injection, oocytes were incubated with ${ }^{35} \mathrm{~S}$-methionine and RINGO/Spy was immunoprecipitated and analyzed by SDS-PAGE. RINGO/Spy protein was syn- thesized only when the Pum2 antibody was injected (Fig. 4D, upper panel, cf. lanes 1 and 4). (Note that in this experiment, the oocytes were also treated briefly with progesterone, which was necessary to detect RINGO/ Spy.) Because several ${ }^{35}$ S-methionine-labeled proteins were immunoprecipitated by the RINGO/Spy antibody, we also injected RINGO/Spy AS ODN to determine whether one specific band was eliminated. Indeed, the AS ODN inhibited the synthesis of the major band that was precipitated by the RINGO/Spy antibody (Fig. 4D, upper panel, lane 6). We also detected RINGO/Spy synthesis as early as $1 \mathrm{~h}$ after hormone treatment in oocytes (Fig. 4D, lower panel, lane 2), as well as in oocytes injected with Pum2 antibody in the absence of any hormone (Fig. 4D, lower panel, lane 6). Thus, we conclude that Pum2 antibody injection induces the synthesis of endogenous RINGO/Spy. As expected, Pum2 antibody injection also accelerated several downstream events that are attributed to RINGO/Spy synthesis including CPEB destruction during M2 (Mendez et al. 2002) and Mos and cyclin B1 synthesis (Fig. 4E; de Moor and Richter 1997).

Finally, the $\mathrm{N}$ terminus of Pum2 as well as the Puf domain were fused to maltose-binding protein (MBP) for synthesis in and purification from E. coli. These proteins, as well as MBP-DAZL as a control, were injected into oocytes that had been injected with RNA encoding GFP-RINGO/Spy-3'UTR (Fig. 4F). While buffer only or MBP-DAZL had little effect on GFP synthesis, the MBPPum2 N terminus (MBP-Pum2-NT) induced the translation of GFP mRNA. The injection of MBP-PUF2 slightly induced GFP synthesis. We therefore conclude that the $\mathrm{N}$-terminal portion of Pum2 acted as a dominant-negative mutant protein to abrogate the activity of endogenous Pum2 and stimulate GFP synthesis. Together, the Pum 2 antibody injection and the Pum 2 dominant-negative mutant protein injection strongly indicate that Pum2 controls the translation of RINGO/SPY mRNA through its $3^{\prime} \mathrm{UTR}$. 
Padmanabhan and Richter

A

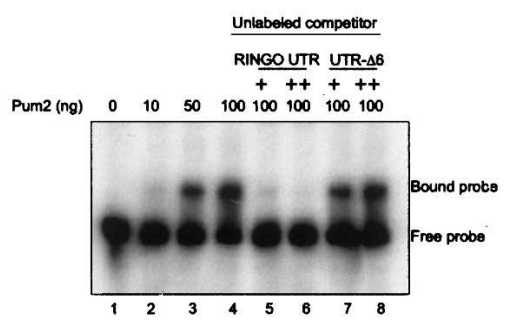

C

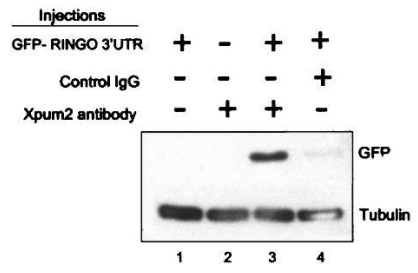

$\mathbf{E}$

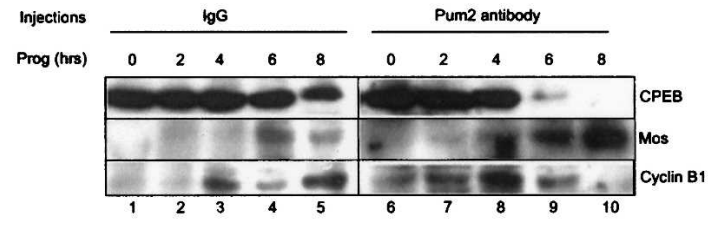

B

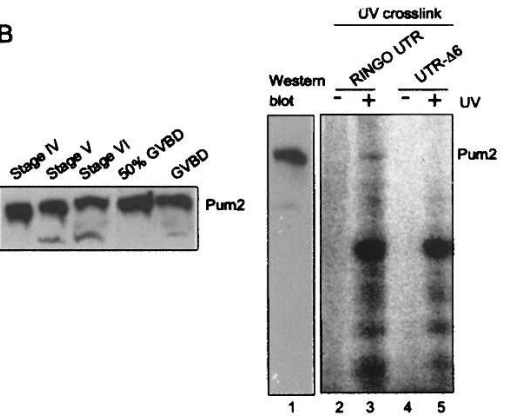

D

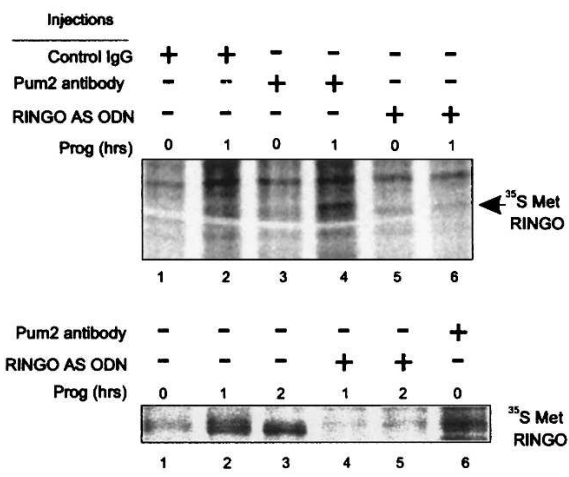

F

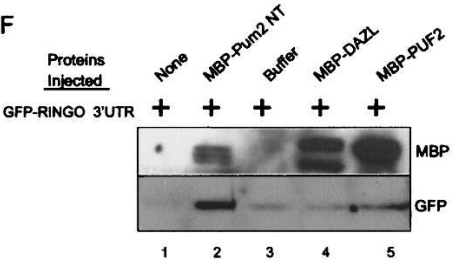

Figure 4. Pumilio 2 binds the RINGO/Spy $3^{\prime} \mathrm{UTR}$ and regulates translation. Various concentrations of recombinant Pum 2 (PUF domain) were mixed with a radiolabeled RINGO/Spy 3'UTR that contained two putative PBEs. In some cases, the mixture also contained excess radio-inert RINGO 3'UTR or the RINGO $\Delta 6$ 3'UTR that lacked both PBEs. The mixtures were applied to a nondenaturing gel, and the free and shifted (bound) RINGO 3'UTR was detected. (B) The left panel is a Western blot for Pum2 that shows that it was present in approximately equal amounts through the later stages of oogenesis as well as during maturation (as scored by germinal vesicle breakdown or GVBD; $50 \%$ GVBD denotes that in this group of oocytes, only half had undergone maturation). The right panels show a UV cross-linking experiment in which a stage VI oocyte extract was supplemented with ${ }^{32} \mathrm{P}-\mathrm{labeled}$ RINGO/Spy full-length 3'UTR or the $\Delta 6$ 3'UTR deletion mutant followed by UV irradiation and RNase treatment. A cross-linked band the size of Pum2 (as compared to a Western blot of oocyte extract) was evident with the full-length 3'UTR but not with the $\Delta 6$ mutant 3'UTR. (C) Oocytes were injected with GFP-RINGO/Spy 3'UTR; following overnight incubation, the oocytes were injected with either purified rabbit IgG, or Pum2 antibody and incubated for a further $10 \mathrm{~h}$. The lysed oocytes were then analyzed on a Western blot for GFP and tubulin as a loading control. $(D)$ Oocytes were injected with either rabbit IgG, Pum2 antibody, or a mix of Pum2 antibody and RINGO AS ODN; they were then cultured in medium containing ${ }^{35} \mathrm{~S}$-methionine $/{ }^{35} \mathrm{~S}$-cysteine mix and in some cases, progesterone as well. (Upper panel) The oocytes were lysed, and RINGO/Spy was immunoprecipitated and analyzed by SDS-PAGE. Other oocytes metabolically labeled with ${ }^{35} \mathrm{~S}$-methionine $/{ }^{35} \mathrm{~S}$-cysteine were incubated for $0-2 \mathrm{~h}$ in progesterone; in one case, the oocytes were also injected with Pum2 antibody (no progesterone, lane 6). (Lower panel) RINGO/Spy was then immunoprecipitated and analyzed by SDS-PAGE. $(E)$ Oocytes were mock-injected or injected with Pum2 antibody, incubated for 3-6 h, and then stimulated with progesterone. The oocytes were collected at several time points, lysed, and probed for CPEB, Mos, and Cyclin B1 on Western blots. $(F)$ The Pum2 N-terminal 310 amino acids (MBP-Pum2 NT) and C-terminal 355 amino acids (MBP-PUF2) were fused to MBP and produced in and isolated from E. coli. MBP fused to DAZL was similarly processed. These proteins $(\sim 50 \mathrm{~nL}$ of solution of $1 \mathrm{mg} / \mathrm{mL})$ were injected into oocytes that were previously injected with mRNA encoding GFP-RINGO/Spy 3'UTR. The amount of MBP fusion protein injected and the amount of GFP produced were assessed by Western blotting. 
Pum2 interacts with DAZL and ePAB but dissociates from RINGO/SPY mRNA during maturation

We next injected oocytes with mRNAs encoding the myc-tagged PUF domain of Pum2; after an overnight incubation, ribonucleoprotein (RNP) complexes were immunoprecipitated with myc antibody and examined for the presence of RINGO/Spy mRNA by RT-PCR. As controls, oocytes were also injected with mRNAs encoding myc-tagged DAZL and myc-tagged CPEB prior to myc IP and RT-PCR. Figure 5A shows that the myc-PUF and myc-DAZL immunoprecipitates contained RINGO/Spy mRNA; however, the myc-CPEB immunoprecipitates contained none of this RNA. Conversely, myc-CPEB immunoprecipitates contained cyclin B1 RNA, an expected result since $\mathrm{CPEB}$ regulates the polyadenylation and translation of this RNA (Stebbins-Boaz et al. 1996). None of the myc immunoprecipitates contained Inh2 RNA, a negative control. These results show that Pum2 and DAZL interact with RINGO/Spy RNA in vivo while CPEB does not.

The interaction of DAZL with RINGO/Spy RNA is perhaps not surprising given that DAZL and Pum2 can bind one another (Moore et al. 2003). DAZL also binds
ePAB, a Xenopus embryonic PABP that stabilizes poly(A) tails on RNAs during maturation and early embryogenesis (Voeltz et al. 2001; Collier et al. 2005). To investigate whether endogenous RINGO/Spy mRNA resides in a complex of Pum2, DAZL, and ePAB, all three proteins were immunoprecipitated from oocytes and examined for the presence of RINGO/Spy RNA by RT-PCR (note that because DAZL antibody does not immunoprecipitate this protein very well, mRNA encoding myc-DAZL was injected into oocytes, followed by myc IP). All three proteins were coimmunoprecipitated with RINGO/Spy RNA; nonspecific IgG did not precipitate RINGO/Spy RNA to an appreciable extent (Fig. 5B, top, cf. lanes 1 and 3). Next, the same IP experiments were performed on oocytes that had been treated with progesterone. Surprisingly, while DAZL and ePAB were still coimmunoprecipitated with RINGO/Spy RNA, Pum2 was not (Fig. 5B, top, cf. lanes 2 and 4). Similar amounts of Pum2, ePAB, and DAZL were immunoprecipitated from untreated or progesterone-treated oocytes (Fig. 5B, bottom). Thus, Pum2 dissociates from RINGO/Spy mRNA during maturation.

The results in Figure 5B also imply that Pum 2 dissociates from DAZL and $\mathrm{PPAB}$ during maturation. To
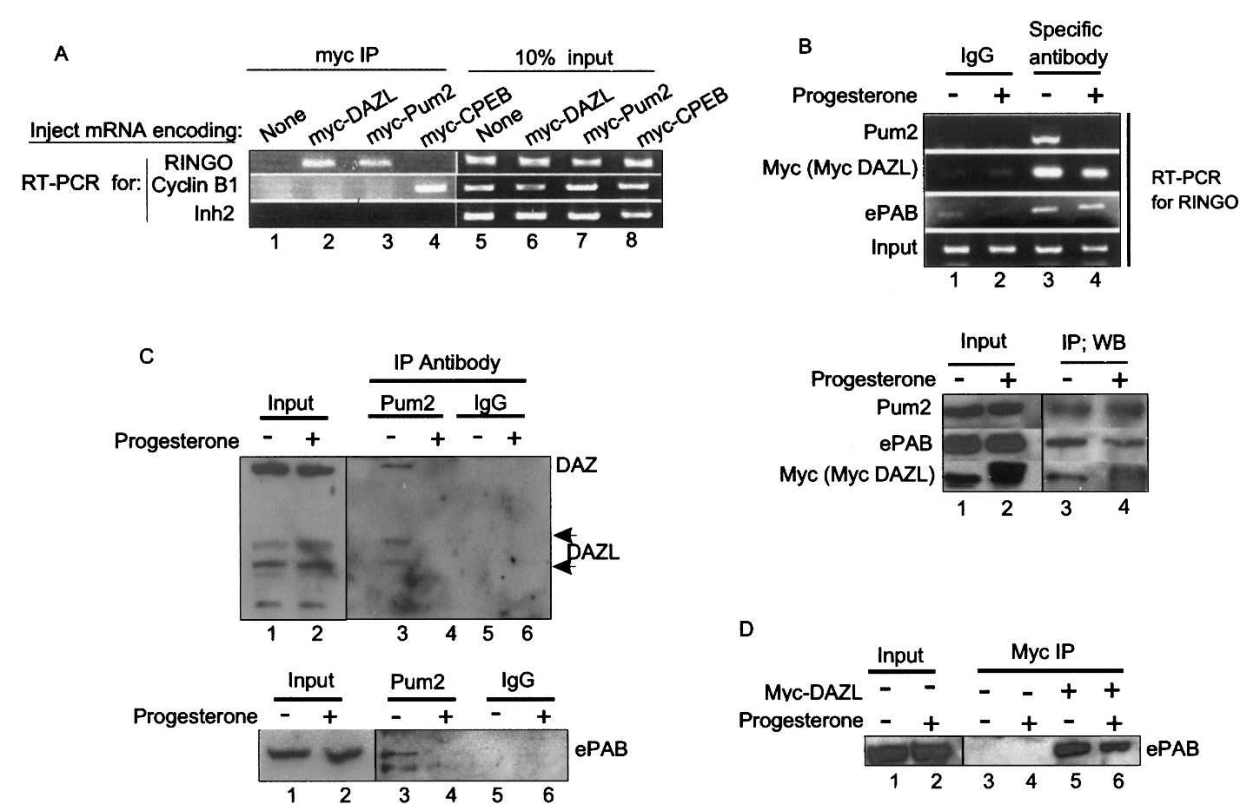

Figure 5. Pum2 binding to RINGO/Spy RNA and DAZL and ePAB is reversible. (A) Oocytes were mock-injected or injected with mRNAs encoding myc-tagged Pum2 (the PUF domain), DAZL, and CPEB and cultured overnight. The oocytes were then lysed for myc IP. RNA was then isolated from the immunoprecipitates and subjected to RT-PCR using specific primers for RINGO/Spy, cyclin B1, and Inh2, an inhibitor of PP1 phosphatase. Also shown are RT-PCR reactions for the RNAs from 10\% of the total lysate. (B) RNPs were immunoprecipitated from control or progesterone-stimulated oocytes by nonspecific IgG or antibodies for Pum2 and ePAB. Because DAZL antibody did not efficiently immunoprecipitate the protein, some oocytes were injected with mRNA encoding myc-DAZL; in this case, myc antibody was used for RNP IP. RNA was extracted from the immunoprecipitates and used to RT-PCR for RINGO/Spy RNA. (Upper panel) RT-PCR was also performed on 10\% of the total lysates. (Lower panel) Western blots were also used to detect Pum2, ePAB, and DAZL from control and progesterone-treated oocytes (input, one oocyte equivalent) and in immunoprecipitates of these proteins (100 oocyte equivalents). (C) Pum2 was immunoprecipitated from control and progesterone-treated oocytes in the presence of RNase A. The immunoprecipitates as well as input lysate (one oocyte) were probed on Western blots for DAZL and ePAB. $(D)$ Oocytes were injected with myc-DAZL mRNA, and myc immunoprecipitates were analyzed for ePAB from control and progesterone-treated oocytes by a Western blot. Input lysates (one oocyte) were also blotted for ePAB. 
assess this possibility, Pum2 was immunoprecipitated (in the presence of RNase A) from untreated and progesterone-stimulated oocytes, followed by Western blotting for DAZL and ePAB. While DAZL (and DAZ, a related protein) was coprecipitated with Pum 2 from oocytes, it was not coprecipitated (nor was DAZ) with Pum2 from progesterone-treated oocytes (Fig. 5C, top). Similarly, ePAB was coprecipitated with Pum2 only in untreated oocytes (Fig. 5C, bottom). However, DAZL and ePAB remained associated irrespective of whether the oocytes were treated with progesterone (Fig. 5D).

Figure 6 presents a model for Pum2-regulated translation of RINGO/Spy mRNA. In oocytes where RINGO/ Spy mRNA is dormant, Pum 2 is bound to the PBE and also interacts with DAZL and ePAB. eIF4E and/or eIF4G may also be associated with the mRNA. Pum2 might repress translation by interrupting or modifying the interactions between the cap and eIF4E, eIF4E and eIF4G, or eIF4G and ePAB. Following progesterone stimulation of maturation, Pum2 dissociates from the RINGO/Spy RNP complex, thereby liberating it for translation.

\section{Discussion}

This study demonstrates that while oocytes do not contain RINGO/Spy protein, an atypical activator of $\operatorname{cdk} 1$, they do contain dormant RINGO/Spy mRNA that is translated soon after the induction of meiotic maturation. RINGO/Spy translation precedes and is necessary for the activation of CPEB by Aurora A-catalyzed S174 phosphorylation and the resulting polyadenylation-induced translation of mos and other mRNAs. Two putative PBEs reside in the RINGO/Spy 3'UTR and are necessary for the repression; they are bound by Pumilio 2 (Pum2), a member of the PUF family of proteins (Wickens et al. 2002). In oocytes, Pum 2 interacts not only with RINGO/Spy RNA, but also with DAZL, an RNA-binding protein, and $\mathrm{PAB}$, the embryonic PABP. In response to progesterone, Pum2 dissociates from this RNP complex, and as a consequence, RINGO/Spy mRNA is translated.

In Xenopus oocytes, the transition from a G2-like arrest to the meiotic divisions is tightly controlled at the level of protein modification (e.g., phosphorylation), synthesis, and destruction. mRNAs such as those encoding Mos are regulated by poly(A) elongation, which is a rela- tively early response to progesterone stimulation that requires a preceding protein synthesis event (McGrew and Richter 1990). That is, oocytes treated simultaneously with CHX and progesterone do not polyadenylate RNA. However, oocytes treated with CHX 60 min or more after progesterone treatment are perfectly competent for polyadenylation (McGrew and Richter 1990). While the nature of the protein(s) whose synthesis was required for polyadenylation remained obscure, three subsequent observations suggested a possible candidate. First, injection of recombinant cyclins reversed the CHX block to polyadenylation (Paris et al. 1991). Second, Nebreda et al. (1995) showed that the activation of MAPK and subsequently of MPF required an early cdk activity. While this early maturation event would not necessarily be linked to or be upstream of polyadenylation, the third observation by Ferby et al. (1999) demonstrating that newly synthesized RINGO/Spy acts as an atypical cyclin cofactor of early cdk activity indicated that it might also be important for polyadenylation. Indeed, the results presented here show that RINGO/Spy mRNA translation is necessary for CPEB phosphorylation and activation. The substrate(s) of RINGO/Spy-cdk activity that promotes CPEB phosphorylation is unknown. While the CPEB phosphorylating enzyme Aurora A is regulated by several factors including TPX2 (Eyers et al. 2003; Tsai et al. 2003) and GSK-3 $\beta$ (Sarkissian et al. 2004), there is no evidence that cdk activity also directly contributes to this regulation. Thus, we surmise that an upstream factor that indirectly influences Aurora A activity is a target of RINGO/Spy-cdk1 activity.

Three lines of evidence point to Pum 2 as the key protein responsible for controlling RINGO/Spy mRNA translation: (1) the injection of neutralizing Pum2 antibody into oocytes stimulates RINGO/Spy synthesis, (2) the injection of a dominant-negative form of Pum2 protein stimulates RINGO/Spy synthesis, and (3) Pum2 dissociates from RINGO/Spy mRNA when its translation is activated. Pum2 is a member of the PUF (Pumilio-FBF) family of RNA-binding proteins (Wickens et al. 2002); the founding member of this family, Pum1, was first shown to be a translational repressor in Drosophila embryos (Murata and Wharton 1995). While there are now known to be multiple PUF proteins in lower eukaryotes (Wickens et al. 2002), vertebrates have only two: Pum1

Figure 6. Model for Pum2 control of RINGO/ Spy mRNA translation. In oocytes, Pum2 binds the PBE and also interacts with DAZL, an RNAbinding protein, and $\mathrm{PAB}$. The RNP may also contain eIF4G, which is important for recruiting the 40s ribosomal subunit to the RNA, or eIF4E, which binds the $5^{\prime}$ cap. Pum2 could repress RINGO/Spy mRNA translation by interfering with or modifying the proper interactions between the cap and eIF4E, between eIF4E and eIF4G, or between eIF4G and ePAB. Following progesterone stimulation of oocyte maturation, Pum2 dissociates from the RINGO/Spy RNP, thereby liberating it for translation.
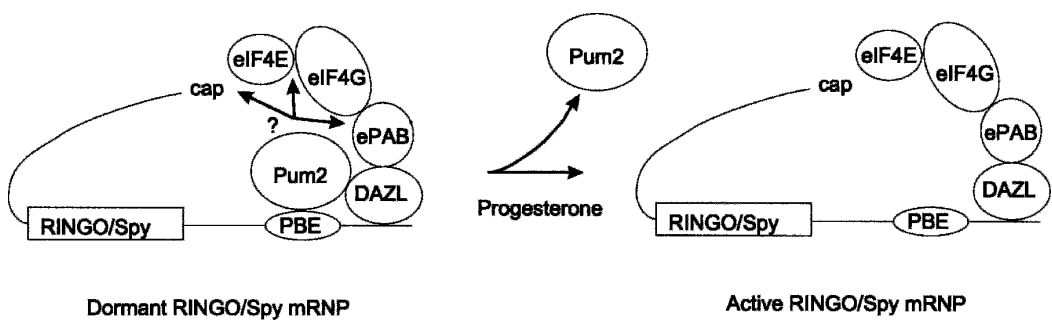
and Pum2. While the classical NRE is the binding site for Pum1, the similar PBE is the binding site for Pum2. Pum1 and Pum2 interact with different proteins that mediate their function as translational regulators. That is, Pum1-mediated translational repression of Hunchback mRNA requires an interaction with the zinc finger protein Nanos (NOS) and the NHL (NCL-1, HT2A, LIN41) (Slack and Ruvkun 1998) domain protein Brain Tumor (BRAT) (Sonoda and Wharton 2001). In vitro, Pum1 interacts with the Nanos homolog XCat2; in Xenopus oocytes, it interacts with CPEB (Nakahata et al. 2001). The significance of these interactions, however, has not been established. Pum2, on the other hand, interacts with human and mouse DAZ and DAZL (Moore et al. 2003). Similarly, in Drosophila, Pum2 interacts with Boule, the DAZ homolog in this species (Urano et al. 2005). In agreement with these findings, our data show that DAZL and Pum2 reside in a complex in Xenopus oocytes. While DAZ and DAZL contain RNA recognition motifs (RRMs), a consensus binding sequence has not been clearly delineated. For example, a G/CU-rich sequence is important for the binding of DAZL to some target RNAs, while a U-rich sequence appears to be important for others (Jiao et al. 2002; Fox et al. 2005). Perhaps a DAZ/DAZL-binding specificity is modulated by interacting factors that differ among cell types.

Recently, Collier et al. (2005) showed that DAZL stimulates translation initiation by interacting with either of two PABPs, "conventional" PABP1 and ePAB. These results suggested that the Pum2-DAZL complex we found in Xenopus would also contain ePAB, the major $\mathrm{PAB}$ in oocytes, which, indeed, was the case. Collier et al. (2005) further suggested that the function of DAZL is to recruit PABP to RNAs and stimulate their translation. In the case we describe here, however, Pum2, DAZL, and ePAB are associated with RINGO/Spy before translation (note that while all three proteins could reside in multiple complexes, it is very likely that all they are simultaneously bound to RINGO/Spy mRNA in oocytes). How could this configuration of proteins repress translation? It would seem unlikely that Pum2 would act at the DAZL-ePAB interface since ePAB is a part of the RNP complex. Perhaps Pum2 inhibits ePAB activity, which, as Collier et al. (2005) point out, could impact eIF4G, eIF4B, or poly(A)-interacting protein (PAIP), which binds the helicase eIF4A. On the other hand, Pum2 might block the interaction of eIF4E with eIF4G like Maskin (Stebbins-Boaz et al. 1999; Cao and Richter 2002), or of eIF4E with the cap like 4EHP (Cho et al. 2005), among other possibilities. Irrespective of the mechanism of Pum2-mediated repression, its dissociation from RINGO/Spy RNA, and from ePAB and DAZL, during maturation is almost certainly necessary for translation. Interestingly, a similar mechanism may be operating in yeast, which contains several PUF proteins (Gerber et al. 2004). Here, PUF6 interacts with the Ash1 RNP complex, which is transported from mother to daughter cell where it controls mating-type switching (Chartrand et al. 2001; Gu et al. 2004). Although Ash1 mRNA is repressed during transport, it is activated once it reaches the bud tip. Yeast strains lacking PUF6 translate Ash1 RNA prematurely, demonstrating its necessity for repression. Gu et al. (2004) thus speculated that PUF6 dissociates from Ash1 following its anchoring at the bud tip, thereby reversing the repression.

Our next goals are to determine how the association of Pum2 with RINGO/Spy mRNA is reversed and how it represses translation. The observation that the injection of the N-terminal portion of Pum2 derepresses RINGO/ Spy translation indicates that it interacts with another regulatory factor in this region, thus providing a possible substrate for the isolation of an associated protein.

\section{Materials and methods}

\section{Oocytes and injections}

Oocytes were isolated from wild-caught animals or lab-bred nonprimed animals (Xenopus One; Nasco Biologicals) and defolliculated using Type II collagenase/Dispase II mix. Analysis of polyadenylation in stage VI Xenopus oocytes has been described (de Moor and Richter 1997). To express heterologous myc-tagged proteins, oocytes were typically injected with $50-\mathrm{nL}$ solutions of RNA $(0.5-1 \mu \mathrm{g} / \mu \mathrm{L})$ and then incubated for $12-18 \mathrm{~h}$, when they were harvested, homogenized in IP buffer containing $150 \mathrm{mM} \mathrm{NaCl}, 50 \mathrm{mM}$ Tris-HCl (pH 7.5), 0.5\% NP-40, $1 \mathrm{mM}$ DTT, $10 \%$ BSA, $0.2 \%$ vanadyl ribonucleoside complex, and RNase OUT, a protease inhibitor cocktail that included $1 \mathrm{mM}$ phenylmethylsulfonylfluoride and $10 \mu \mathrm{g} / \mathrm{mL}$ each leupeptin, chymostatin, pepstatin, and aprotinin. In some cases, RNase A (50 $\mathrm{\mu g} / \mathrm{mL}$ final concentration) was added to the IP buffer. To inject recombinant proteins, $50-\mathrm{nL}$ solutions containing $0.5-1$ $\mu \mathrm{g} / \mu \mathrm{L}$ concentrations were used; if the proteins were stored in glycerol, it was diluted so that it was $\leq 1 \%$.

\section{Cloning and expression of recombinant proteins}

The Xenopus Pum2-coding region was amplified and spliced from three separate PCR reactions, the first two of which were derived from IMAGE clone 6633118 and IMAGE clone 5156081. The third reaction to obtain the $\mathrm{N}$-terminal 30 amino acids was derived from a PCR reaction using degenerate primers based on a Xenopus tropicalis Pum2 EST sequence and specific reverse primers based on sequence obtained from IMAGE clone 6633118. The spliced product was cloned into pET30a and pET30a-Myc vectors. The 3'UTRs of RINGO/Spy were cloned into the HindIII-NotI sites of pBluescriptSK- ${ }^{-}$RNAs were synthesized from templates linearized by NotI, using mMessage mMachine transcription kits from Ambion. The coding portion of Xenopus RINGO/Spy was amplified from total RNA and cloned into pET30a, pMALC2, and pBluescript vectors. Xenopus DAZL was amplified from total RNA and cloned into pBluescript and pET30a vectors using EcoRI and XhoI. For protein expression, plasmids were transfected into BL21 DE3 cells and then induced with $1 \mathrm{mM}$ IPTG. MBP-RINGO/Spy and other MBP-tagged proteins were purified as described in Ferby et al. (1999).

\section{Antibodies}

Recombinant RINGO/Spy was used to immunize rabbits to obtain anti-serum. Antibodies for Mos (Santa Cruz), myc (9E10), GFP (Molecular Probes), and human Pum2 (Bethyl Labs) were obtained from commercial sources. CPEB antibody has been 
described (Hake and Richter 1994). Antibody that recognized Xenopus DAZL (and DAZ) was a generous gift from Dr. Renee Reijo Pera (University of California at San Francisco, San Francisco, CA). Antibody for ePAB was a generous gift from Dr. Joan Steitz (Yale Medical School, New Haven, CT).

Electrophoretic gel mobility-shift assays, UV cross-linking, and RNA affinity

The RNA-binding domain (PUF domain) of Pum2 was cloned into pET30a and used to generate recombinant protein in E. coli. Proteins were purified and dialyzed against buffer containing 10 mM HEPES (pH 7.5), 50 mM KCl, 2 mM DTT, $0.1 \mathrm{mg} / \mathrm{mL}$ BSA, $0.01 \%$ Tween 20 , and $1 \mathrm{mM}$ EDTA as described in Zamore et al. (1997) and Murata and Wharton (1995). Electrophoretic mobility-shift assays (EMSA) were performed essentially as described by Zamore et al. (1997). UV cross-linking assays were performed as described by Hake and Richter (1994).

\section{IPs and Western blots}

To immunoprecipitate Pum2, DAZL, and ePAB for analysis by Western blotting, oocytes were homogenized in $20 \mathrm{mM}$ sodium phosphate buffer ( $\mathrm{pH} 7.6)$ containing $150 \mathrm{mM} \mathrm{NaCl}, 0.25 \%$ NP40, 10\% glycerol, $1 \mathrm{mM}$ DTT, $2 \mathrm{mM}$ EDTA, $1 \mathrm{mM} \beta$-mercaptoethanol, $1 \mathrm{mM} \mathrm{MgCl}, 1 \mathrm{mM}$ PMSF, $50 \mu \mathrm{g} / \mathrm{mL}$ RNase A, and protease inhibitor cocktail (Roche). After a clarifying centrifugation, the extracts were incubated with $2-4 \mu \mathrm{L}$ of immune serum or IgG for $2 \mathrm{~h}$ at $4^{\circ} \mathrm{C}$. Fifteen microliters of magnetic beads conjugated with anti-rabbit IgG (Dyna Beads; Dynal Biotech) were added, and the mixture was further incubated for another $2 \mathrm{~h}$ with gentle mixing at $4^{\circ} \mathrm{C}$. The beads were then washed with $4 \mathrm{~mL}$ of cold homogenization buffer and finally boiled in SDS sample buffer containing 50 mM DTT. Primary antibodies were used at a dilution of 1:1000. Mouse or rabbit TruBlot secondary antibodies (eBioscience) were used as secondary antibodies for appropriate experiments followed by ECL detection using the Super Signal West Dura kit from Pierce.

For RINGO/Spy IP, oocytes were first lysed in $100 \mu \mathrm{L}$ of buffer (150 mM NaCl, $50 \mathrm{mM}$ Tris- $\mathrm{HCl}$ at $\mathrm{pH}$ 7.5, 0.1\% NP-40, protease inhibitor cocktail [Roche], $1 \mathrm{mM}$ PMSF) and precleared before the lysate was diluted to $500 \mu \mathrm{L}$ in RIPA buffer $(50 \mathrm{mM}$ Tris- $\mathrm{HCl}$ at $\mathrm{pH} 7.4,150 \mathrm{mM} \mathrm{NaCl}, 0.1 \%$ SDS, $1 \% \mathrm{NP}-40$, and protease inhibitor cocktail). The mixture was then added to protein A Sepharose beads and incubated for $4-12 \mathrm{~h}$ at $4^{\circ} \mathrm{C}$. The beads were washed extensively with RIPA buffer before boiling in SDS sample buffer and Western blotting.

To radiolabel oocytes with ${ }^{35} \mathrm{~S}$-methionine $/{ }^{35} \mathrm{~S}$-cysteine, oocytes were incubated in medium containing $2 \mathrm{mCi} / \mathrm{mL}^{35} \mathrm{~S}$ Promix (Amersham) following injections of AS ODNs and antibodies. Progesterone was added to oocytes $5 \mathrm{~h}$ after AS ODN injections. RINGO IPs were then performed as described above. For ${ }^{32} \mathrm{P}$ labeling of endogenous CPEB, oocytes were injected with solution of $3000 \mathrm{Ci} / \mathrm{mmol}\left[\gamma_{-}{ }^{32} \mathrm{P}\right] \mathrm{ATP}$, followed by progesterone stimulation and collection. Endogenous CPEB was immunoprecipitated as described in Mendez et al. (2000).

For IPs followed by RNA analysis, 50-100 oocytes were typically used. CPEB was immunoprecipitated as described by Mendez et al. (2000). Pum2, both endogenous and myc-tagged forms, was immunoprecipitated from oocytes homogenized in 150 $\mathrm{mM} \mathrm{NaCl}, 50$ mM Tris- $\mathrm{HCl}$ (pH 7.5), 0.5\% NP-40, 1 mM DTT, $10 \%$ BSA, vanadyl ribonucleoside complex, RNase OUT, and protease inhibitor cocktail that included $1 \mathrm{mM}$ phenylmethylsulfonylfluoride and $10 \mu \mathrm{g} / \mathrm{mL}$ each leupeptin, pepstatin, chymostatin, and aprotinin. The extracts were precleared using Sepharose beads for $30 \mathrm{~min}$ at $4^{\circ} \mathrm{C}$. The antibody was then added, and the mixture was incubated for $2 \mathrm{~h}$ at $4^{\circ} \mathrm{C}$, after which it was washed with $2-3 \mathrm{~mL}$ of homogenization buffer. RNA was extracted from the beads, and RT-PCR was performed as described by Tay and Richter (2001).

\section{Acknowledgments}

We thank Dr. J. Steitz (Yale Medical School) and Dr. R. Reijo Pera (University of California at San Francisco) for gifts of antibodies. This work was supported by a grant from the NIH (GM46779). Additional support from the Diabetes Endocrinology Research Center Program Project (DK32520) is also gratefully acknowledged.

\section{References}

Ballantyne, S., Daniel Jr., D.L., and Wickens, M. 1997. A dependent pathway of cytoplasmic polyadenylation reactions linked to cell cycle control by c-mos and CDK1 activation. Mol. Biol. Cell 8: 1633-1648.

Barnard, D.C., Ryan, K., Manley, J.L., and Richter, J.D. 2004. Symplekin and xGLD-2 are required for CPEB-mediated cytoplasmic polyadenylation. Cell 119: 641-651.

Barnard, D.C., Cao, Q., and Richter, J.D. 2005. Differential phosphorylation controls Maskin association with eukaryotic translation initiation factor $4 \mathrm{E}$ and localization on the mitotic apparatus. Mol. Cell. Biol. 25: 7605-7615.

Cao, Q. and Richter, J.D. 2002. Dissolution of the maskin-eIF4E complex by cytoplasmic polyadenylation and poly(A)-binding protein controls cyclin B1 mRNA translation and oocyte maturation. EMBO J. 21: 3852-3862.

Chartrand, P., Singer, R.H., and Long, R.M. 2001. RNP localization and transport in yeast. Ann. Rev. Cell. Dev. Biol. 17: 297-310.

Cho, P.F., Poulin, F., Cho-Park, Y.A., Cho-Park, I.B., Chicoine, J.D., Lasko, P., and Sonenberg, N. 2005. A new paradigm for translational control: Inhibition via $5^{\prime}-3^{\prime}$ mRNA tethering by Bicoid and the eIF4E cognate 4EHP. Cell 121: 411-423.

Collier, B., Gorgoni, B., Loveridge, C., Cooke, H.J., and Gray, N.K. 2005. The DAZL family proteins are PABP-binding proteins that regulate translation in germ cells. EMBO $J$. 24: 2656-2666.

Cooke, H.J. and Elliott, D.J. 1997. RNA-binding proteins and human male infertility. Trends Genet. 13: 87-89.

de Moor, C.H. and Richter, J.D. 1997. The Mos pathway regulates cytoplasmic polyadenylation in Xenopus oocytes. Mol. Cell. Biol. 17: 6419-6426.

Dinarina, A., Perez, L.H., Davila, A., Schwab, M., Hunt, T., and Nebreda, A.R. 2005. Characterization of a new family of cyclin-dependent kinase activators. Biochem. J. 386: 349-355.

Eyers, P.A., Erikson, E., Chen, L.G., and Maller, J.L. 2003. A novel mechanism for activation of the protein kinase Aurora A. Curr. Biol. 13: 691-697.

Ferby, I., Blazquez, M., Palmer, A., Eritja, R., and Nebreda, A.R. 1999. A novel p34(cdc2)-binding and activating protein that is necessary and sufficient to trigger $G(2) / M$ progression in Xenopus oocytes. Genes \& Dev. 13: 2177-2189.

Ferrell Jr., J.E. 1999. Xenopus oocyte maturation: New lessons from a good egg. Bioessays 21: 833-842.

Fox, M., Urano, J., and Reijo Pera, R.A. 2005. Identification and characterization of RNA sequences to which human PUMILIO-2 (PUM2) and deleted in Azoospermia-like (DAZL) bind. Genomics 85: 92-105.

Gerber, A.P., Herschlag, D., and Brown, P.O. 2004. Extensive 
association of functionally and cytotopically related mRNAs with Puf family RNA-binding proteins in yeast. PLoS Biol. 2: E79.

Gu, W., Deng, Y., Zenklusen, D., and Singer, R.H. 2004. A new yeast PUF family protein, Puf6p, represses ASH1 mRNA translation and is required for its localization. Genes \& Dev. 18: $1452-1465$.

Hake, L.E. and Richter, J.D. 1994. CPEB is a specificity factor that mediates cytoplasmic polyadenylation during Xenopus oocyte maturation. Cell 18: 617-627.

Jiao, X., Trifillis, P., and Kiledjian, M. 2002. Identification of target messenger RNA substrates for the murine deleted in azoospermia-like RNA-binding protein. Biol. Reprod. 66: 475-485.

Karaiskou, A., Perez, L.H., Ferby, I., Ozon, R., Jessus, C., and Nebreda, A.R. 2001. Differential regulation of Cdc2 and Cdk2 by RINGO and cyclins. I. Biol. Chem. 276: 3602836034.

Lenormand, J.L., Dellinger, R.W., Knudsen, K.E., Subramani, S., and Donoghue, D.J. 1999. Speedy: A novel cell cycle regulator of the G2/M transition. EMBO J. 18: 1869-1877.

McGrew, L.L. and Richter, J.D. 1990. Translational control by cytoplasmic polyadenylation during Xenopus oocyte maturation: Characterization of cis and trans elements and regulation by cyclin/MPF. EMBO J. 9: 3743-3751.

Mendez, R., Hake, L.E., Andresson, T., Littlepage, L.E., Ruderman, J.V., and Richter, J.D. 2000. Phosphorylation of CPE binding factor by Eg2 regulates translation of c-mos mRNA. Nature 404: 302-307.

Mendez, R., Barnard, D., and Richter, J.D. 2002. Differential mRNA translation and meiotic progression require Cdc2mediated CPEB destruction. EMBO J. 21: 1833-1844.

Moore, F.L., Jaruzelska, J., Fox, M.S., Urano, J., Firpo, M.T., Turek, P.J., Dorfman, D.M., and Pera, R.A. 2003. Human Pumilio-2 is expressed in embryonic stem cells and germ cells and interacts with DAZ (Deleted in AZoospermia) and DAZ-like proteins. Proc. Natl. Acad. Sci. 100: 538-543.

Murata, Y. and Wharton, R.P. 1995. Binding of pumilio to maternal hunchback mRNA is required for posterior patterning in Drosophila embryos. Cell 80: 747-756.

Nakahata, S., Katsu, Y., Mita, K., Inoue, K., Nagahama, Y., and Yamashita, M. 2001. Biochemical identification of Xenopus Pumilio as a sequence-specific cyclin B1 mRNA-binding protein that physically interacts with a Nanos homolog, Xcat-2, and a cytoplasmic polyadenylation element-binding protein. J. Biol. Chem. 276: 20945-20953.

Nebreda, A.R., Gannon, J.V., and Hunt, T. 1995. Newly synthesized protein(s) must associate with p34cdc2 to activate MAP kinase and MPF during progesterone-induced maturation of Xenopus oocytes. EMBO J. 14: 5597-5607.

Paris, J., Swenson, K., Piwnica-Worms, H., and Richter, J.D. 1991. Maturation-specific polyadenylation: In vitro activation by $\mathrm{p} 34 \mathrm{cdc} 2$ and phosphorylation of a 58-kD CPE-binding protein. Genes \& Dev. 5: 1697-1708.

Porter, L.A., Kong-Beltran, M., and Donoghue, D.J. 2003. Spy 1 interacts with $\mathrm{p} 27 \mathrm{Kip} 1$ to allow G1/S progression. Mol. Biol. Cell 14: 3664-3674.

Sagata, N., Oskarsson, M., Copeland, T., Brumbaugh, J., and Vande Woude, G.F. 1988. Function of c-mos proto-oncogene product in meiotic maturation in Xenopus oocytes. Nature 335: 519-525.

Sarkissian, M., Mendez, R., and Richter, J.D. 2004. Progesterone and insulin stimulation of CPEB-dependent polyadenylation is regulated by Aurora A and glycogen synthase kinase-3. Genes \& Dev. 18: 48-61.

Sheets, M.D., Fox, C.A., Hunt, T., Vande Woude, G., and Wick- ens, M. 1994. The 3'-untranslated regions of c-mos and cyclin mRNAs stimulate translation by regulating cytoplasmic polyadenylation. Genes \& Dev. 8: 926-938.

Slack, F.J. and Ruvkun, G. 1998. A novel repeat domain that is often associated with RING finger and B-box motifs. Trends Biochem. Sci. 23: 474-475.

Sonoda, J. and Wharton, R.P. 2001. Drosophila Brain Tumor is a translational repressor. Genes \& Dev. 15: 762-773.

Stebbins-Boaz, B., Hake, L.E., and Richter, J.D. 1996. CPEB controls the cytoplasmic polyadenylation of cyclin, Cdk2 and c-mos mRNAs and is necessary for oocyte maturation in Xenopus. EMBO J. 15: 2582-2592.

Stebbins-Boaz, B., Cao, Q., de Moor, C.H., Mendez, R., and Richter, J.D. 1999. Maskin is a CPEB-associated factor that transiently interacts with elF-4E. Mol. Cell. 4: 1017-1027.

Tay, J. and Richter, J.D. 2001. Germ cell differentiation and synaptonemal complex formation are disrupted in CPEB knockout mice. Dev. Cell 1: 201-213.

Tsai, M.Y., Wiese, C., Cao, K., Martin, O., Donovan, P., Ruderman, J., Prigent, C., and Zheng, Y. 2003. A Ran signaling pathway mediated by the mitotic kinase Aurora A in spindle assembly. Nat. Cell Biol. 5: 242-248.

Urano, J., Fox, M.S., and Reijo Pera, R.A. 2005. Interaction of the conserved meiotic regulators, BOULE (BOL) and PUMILIO-2 (PUM2). Mol. Reprod. Dev. 71: 290-298.

Voeltz, G.K., Ongkasuwan, J., Standart, N., and Steitz, J.A. 2001. A novel embryonic poly(A) binding protein, ePAB, regulates mRNA deadenylation in Xenopus egg extracts. Genes \& Dev. 15: 774-788.

White, E.K., Moore-Jarrett, T., and Ruley, H.E. 2001. PUM2, a novel murine puf protein, and its consensus RNA-binding site. RNA 7: 1855-1866.

Wickens, M., Bernstein, D.S., Kimble, J., and Parker, R. 2002. A PUF family portrait: 3'UTR regulation as a way of life. Trends Genet. 18: 150-157.

Zamore, P.D., Williamson, J.R., and Lehmann, R. 1997. The Pumilio protein binds RNA through a conserved domain that defines a new class of RNA-binding proteins. RNA 3: 1421-1433.

Zamore, P.D., Bartel, D.P., Lehmann, R., and Williamson, J.R. 1999. The PUMILIO-RNA interaction: A single RNA-binding domain monomer recognizes a bipartite target sequence. Biochemistry 38: 596-604. 


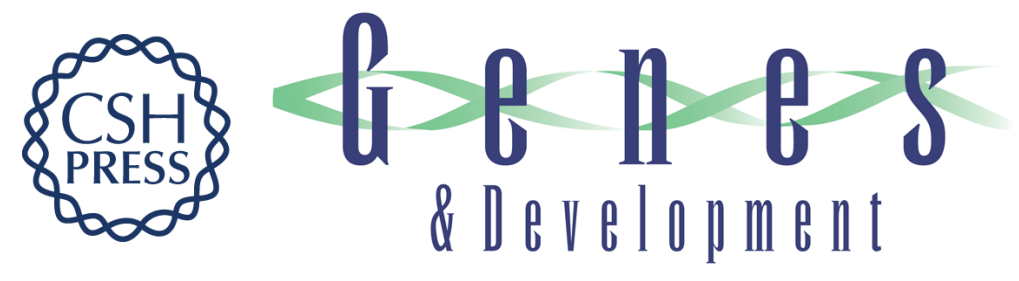

\section{Regulated Pumilio-2 binding controls RINGO/Spy mRNA translation and CPEB activation}

Kiran Padmanabhan and Joel D. Richter

Genes Dev. 2006, 20:

Access the most recent version at doi:10.1101/gad.1383106
Related Content Metazoan oocyte and early embryo development program: a progression through translation regulatory cascades
Shobha Vasudevan, Emre Seli and Joan A. Steitz
Genes Dev. January, 2006 20: 138-146
References This article cites 44 articles, 19 of which can be accessed free at:
http://genesdev.cshlp.org/content/20/2/199.full.html\#ref-list-1
Articles cited in:
http://genesdev.cshlp.org/content/20/2/199.full.htm|\#related-urls

\section{License}
Email Alerting
Receive free email alerts when new articles cite this article - sign up in the box at the top Service

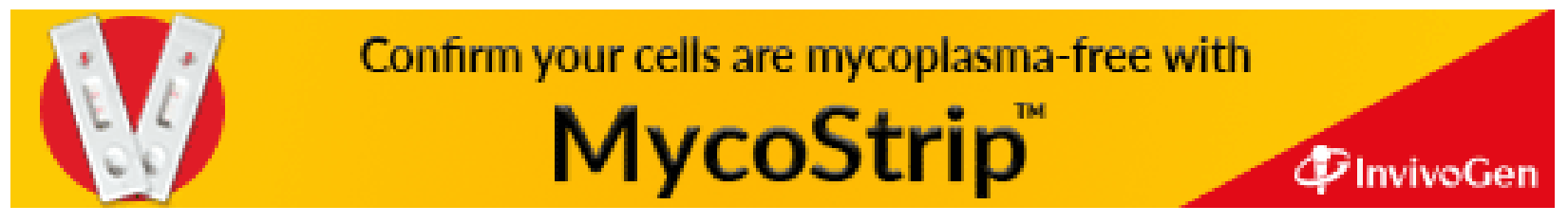

